\title{
Intentionality and "free-will" from a neurodevelopmental perspective
}

\author{
Gerry Leisman $^{1,2,3,4 *}$, Calixto Machado ${ }^{4}$, Robert Melillo ${ }^{1,2,3}$ and Raed Mualem ${ }^{1,3}$ \\ ${ }^{1}$ National Institute for Brain and Rehabilitation Sciences, Nazareth, Israel \\ ${ }^{2}$ F. R. Carrick Institute for Clinical Ergonomics, Rehabilitation, and Applied Neuroscience, Garden City, NY, USA \\ ${ }^{3}$ Nazareth Academic Institute, Nazareth, Israel \\ ${ }^{4}$ Department of Clinical Neurophysiology, Instituto de Neurología y Neurocirugía, La Habana, Cuba
}

\section{Edited by:}

Jose L. Perez Velazquez, Hospital for

Sick Children and University of

Toronto, Canada

\section{Reviewed by:}

Antonio Pereira, Federal University of Rio Grande do Norte, Brazil

Russell Schachar, The Hospital for

Sick Children, Canada

\section{*Correspondence:}

Gerry Leisman, National Institute for

Brain and Rehabilitation, $P O$ Box

50228, Schneler 906/4, Nazareth

16100, Israel.

e-mail:gerry.leisman@

staff.nazareth.ac.il
The nature of free-will as a subset of intentionality and probabilistic and deterministic function is explored with the indications being that human behavior is highly predictable which in turn, should compromise the notion of free-will. Data supports the notion that age relates to the ability to progressively effectively establish goals performed by fixed action patterns and that these FAPs produce outcomes that in turn modify choices (free-will) for which FAPs need to be employed. Early goals require behaviors that require greater automation in terms of FAPs that lead to goals being achieved or not; if not, then one can change behavior and that in turn is free-will. Goals change with age based on experience which is similar to the way in which movement functions. We hypothesize that human prefrontal cortex development was a natural expansion of the evolutionarily earlier developed areas of the frontal lobe and that goal-directed movements and behavior, including choice and free-will, provided for an expansion of those areas. The same regions of the human central nervous system that were already employed for better control, coordination, and timing of movements, expanded in parallel with the frontal cortex. The initial focus of the frontal lobes was the control of motor activity, but as the movements became more goal-directed, greater cognitive control over movement was necessitated leading to voluntary control of FAPs or free-will. The paper reviews the neurobiology, neurohistology, and electrophysiology of brain connectivities developmentally, along with the development of those brain functions linked to decision-making from a developmental viewpoint. The paper reviews the neurological development of the frontal lobes and inter-regional brain connectivities in the context of optimization of communication systems within the brain and nervous system and its relation to free-will.

Keywords: free-will, functional connection, fixed-action patterns, self-regulation, frontal-lobe, goal direction, electrophysiology

\section{THE DEVELOPMENT OF SELF-REGULATION AND TEMPERAMENT}

A two-year-old discovering something that resembles independence by saying "no" is exercising his free-will but for the fact that it is a natural consequence of universal child development, and, therefore a consequence not of variations of child rearing practices but rather of built-in component processing that consequentially results in highly predictable behavioral patterns. We know that some actions are freely chosen and others are automatic reactions. We should be able to characterize the difference between them. We know that natural phenomena are caused by other natural phenomena. The activity of the human mind has been consistently shown to depend on the functioning of the human brain and the latter consists of natural phenomena involving neurons, action potentials, neurotransmitters, and so on. There is no reason to suppose that the causal chains that determine neural events are broken at some point. There is no need to deny that free-will exists, since we need this concept to distinguish between human actions. Therefore, we must understand free-will as a part of the workings of the human brain. We should change our concept of freedom, so as to preserve what is essential in it, eliminating the idea that freedom must escape natural causality. An action is free when it results from a conscious intention to start it, continue it, or at least not to refrain from starting or continuing it. There is no need to suppose that this intention must not be the result of causal processes in the brain. A person is responsible for an action when this action results from a conscious intention. To be responsible for an action is not to be ultimately responsible for it, in the sense of also being responsible for all the events in the causal chains that led to the existence of the conscious intention that determined the action. The nature of the neurological components of behavioral independence requires examination in the context of the well-debated, but yet unresolved nature of free-will.

One concept that mediates the behavior of free-will is the notion of self-regulation and that has been commonly described developmentally as temperament, mediated in turn by frontal 
lobe function (cf. Leisman and Melillo, 2012). Temperament, in the context of frontal lobe development, refers to individual differences in motor and emotional reactivity and self-regulation according to Rothbart and Bates (1998). The temperamental variable related to the development of executive attention is termed effortful control, representing the infant's ability to inhibit a dominant response in order to perform a sub-dominant response. The construct of effortful control is extremely important in understanding the nature of intentionality and free-will.

Most of the more behaviorally based definitions of temperament have focused on temperament's more reactive aspects related to positive and negative affect, reward, punishment, and arousal to stimulation in the development of effortful control of the child's behavior. The processes underlying this effortful control and hence free-will are critical to socialization and have been studied by many. Kochanska (1995), for example, has had indicated that free-will is related to temperamental individual differences in effortful control. He examined longitudinally the development of the complex relation between compliance and internalization in childhood. He distinguished between two forms that he measured, when the child eagerly embraced and endorsed the mother's agenda, and situational, when the child was cooperative, but lacked the sincere commitment and feeling of internal obligation. This psych-social aspect of child development is not the focus of this paper and hence will not be expanded upon here. Brain-based systems of effortful control and temperament instead largely focus on stimulation and either approach or avoidance responses to that stimulation.

Although the literature on temperament had originally reflected the notion that temperament systems would be in place very early in development and change little over time (Buss and Plomin, 1975; Rowe and Plomin, 1977) we have since learned that temperament systems follow a developmental course. Infant's reactive tendencies to experience and their emotional expressivity and response to events in their environment can be observed very early in life. Children's self-regulatory executive attention develops relatively late and coincidently with the development of their frontal and prefrontal cortices throughout the early school years.

Self-regulation, a direct manifestation of temperament, involves complex questions about the nature of volition and its relation to our genetic endowment and to social experiences. Much of the work on self-regulation has been purely behavioral. The lack of appropriate methods to study the physiology of the developing human brain has led to understandable hesitation in thinking about these processes at the neuro-systems level. Kandel (1999) however, has argued persuasively that new concepts in the neurosciences make it possible to relate higher level cognitive concepts to underlying brain systems.

\section{SELF-REGULATION AND THE CONTROL OF DISTRESS IN THE INFANT}

Control of distress is a major task for the infant and caregiver in the early months of life and of course the tasks involved in distress control in turn require external or self-regulatory processes. In the first few months, caregivers help control distress mainly by holding and rocking. Increasingly in the early months, visual orienting is also used. Caregivers then attempt to involve the child in activities that will occupy his or her attention and reduce distress. These interactions between infant and caregiver may train the infant in the control of distress and lead to the development of the mid-frontal area as a control system for negative emotion. Later when similar cognitive challenges arise, a system for regulating remote brain areas may already be prepared.

Evidence exists for a physiological basis of individual differences in the self-regulation of distress and its resultant system of emotional control (inhibition) in human infants. Differences among infants in negative and positive emotionality are often assumed to reflect differences in temperament and are expected to have a constitutional basis (Goldsmith et al., 1987; Porges and Furman, 2011). According to Rothbart (1989), the constitutional basis of temperament should be reflected in endocrine processes that may, in turn, be related to genetic differences among individuals (Buss and Plomin, 1986; Pluess and Belsky, 2011).

We can see in the patterns of resting frontal EEG of neonates and infants significant individual differences of asymmetry that tend to remain in a stable fashion across development of the child and adolescent (Henderson et al., 2001). Davidson and Fox (1989) examined whether certain features of infant temperament might be related to individual differences in the asymmetry of resting frontal activation. EEG was recorded from left and right frontal and parietal scalp regions of 13 normal 10-month-old infants. Infant behavior was then observed during a brief period of maternal separation. Those infants who cried in response to maternal separation showed greater right frontal activation during the preceding baseline period, as exemplified in Figure 1 below, compared with infants who did not cry. Frontal activation

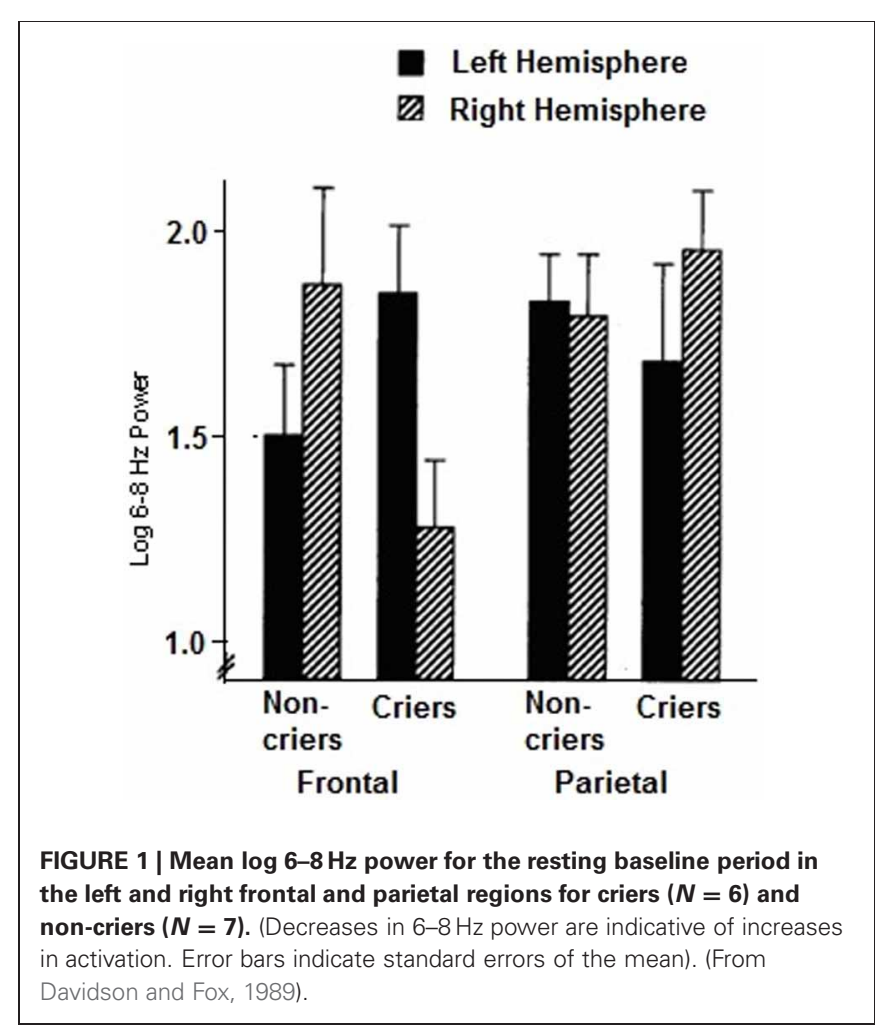


asymmetry may be a state-independent marker for individual differences in the threshold of reactivity to stressful events.

Davidson (2000) had reported that left frontal EEG asymmetry at rest is associated with differential abilities of neonates to regulate their own behavior. Fox and colleagues have indicated that infants displaying elevated left frontal EEG asymmetry at rest exhibit "easy" temperaments, manifested by reports of these infants being easily soothed and calmed.

In contrast, Davidson and Fox (1989) noted that negative affect and a lowered ability by the infant to regulate his or her affect and behavior is highly associated with lower right frontal EEG asymmetry at rest. Infants, when crying demonstrated elevated right frontal activity during the baseline conditions preceding crying. This is highly associated with what we have reported elsewhere in asymmetric activation patterns in ADHD and autistic spectrum children (Leisman and Melillo, 2006; Melillo and Leisman, 2009a,b). Fox reports that the infant's who exhibited this pattern of right frontal EEG asymmetry in the resting state, can be characterized as having "negative reactive" temperaments (Fox et al., 2001). These infants are easily distressed, are difficult to soothe, do not generally self-soothe, and have problems switching and focusing attention. Although the examination of neuroelectrical activity is promising with respect to identifying individual infants with immature or defective neuro-integration or organization, the examination is limited by our imperfect understanding of the relationship between such neuroelectrical activity and behavior at the present time.

Hyperactivity, depression, poor sustained attention, language difficulties, and impulsive or impetuous behavior are common in children with inadequately developing frontal lobe function (Leisman and Melillo, 2012). Children with these behaviors likewise have difficulty being able to self-soothe and a difficulty in impulse control which compromises free-will. These behaviors, when seen later in middle childhood and beyond, are characterized as abnormal. When observed in the neonate and in infancy, these behaviors are seen as normal. In both of the aforementioned cases, immaturity of or compromise to the adequate development of the frontal lobes affects the development of free-will.

\section{FRONTAL LOBES AND AFFECT REGULATION IN THE DEVELOPMENT OF FREE-WILL \\ FRONTAL LOBES AND MOTOR CONTROL AS AN EVOLUTIONARY BASIS OF COGNITION AND BEHAVIORAL INHIBITION}

Major developmental events in the first year include the cortical inhibition of the brainstem, the improvement in recognition and working memory, and the appearance of separation anxiety. From around the age of 3 months, the neonatal palmer grasp reflex begins to disappear. This event has long been known to be related in time to the differentiation of the pyramidal neurons in the supplementary motor cortex (Kennard et al., 1934). Stimuli are transmitted by the cortico-bulbar tract to the interneurons in the brainstem. These inhibit the motor neurons by means of the neurotransmitter GABA, leading to an inhibition of the reflex action of the muscles of the hand. At this age, the cortico-bulbar tract shows intensive myelination, accelerating nerve conduction speed. In the brainstem, synaptic contacts of interneurons to motor neurons are intensified, and GABA synthesis is increased.
These processes increase the cortical inhibition of brainstem reflex activity. If brainstem mechanisms controlling respiration are immature or compromised, then the increased cortical inhibition could place infants at risk for sudden infant death syndrome, which can occur around that age (Touwen, 1971; Wiemann et al., 2008). Conversely, persisting primitive brainstem reflex activity may be associated with a lack of cortical inhibition.

The frontal lobe (Figure 2) plays a major role in motor activities like planning and in the execution of movements. The primary motor area proximal to the precentral gyrus is known as the motor strip (Brodmanns area 4). This is located just anterior to the central sulcus. The primary motor area is also referred to as motor area 1 or MI. Anterior to this area are two additional primary motor areas (Brodmann's 4, 5, and 6). This supplementary motor cortex lies anterior to the motor strip and extends around to the hemisphere's medial surface. The premotor cortex lies anterior to the supplementary motor cortex and on the lateral surface of the hemispheres. These motor areas contain motor neurons whose axons extend to the spinal cord and brainstem and synapse on motor neurons in the spinal cord. The motor neurons are located in layer 5 , the output layer of the motor cortex. This layer contains large pyramidal cells; they are the largest neurons in the cerebral cortex. The most anterior region of the frontal lobe, the prefrontal cortex is responsible for higher aspects of motor control and planning and in the execution of behavior, all tasks requiring integration of information over time. The prefrontal cortex has two main areas, the dorsolateral prefrontal cortex, which is found on the lateral surface of the frontal lobe anterior to the premotor regions, and the orbitofrontal cortex. The orbitofrontal cortex is located on the frontal lobe's anteriorventral surface and is more medial. The orbitofrontal cortex includes limbic lobe structures and is connected to them.

The frontal lobe is the largest lobe in humans (Figure 2), represents one-third of the neocortex and the prefrontal cortex constitutes approximately $50 \%$ of the size of the frontal lobes. The prefrontal cortex is included in a neuronal system that includes the basal ganglia, the thalamus, and the cerebellum. Most of the higher and more complex motor, cognitive, and emotional behavioral functions are thought to be found primarily in the frontal lobes. This area of the neocortex has expanded evolutionarily more than any other in the human brain.

The prefrontal cortex is unique to humans; the reference to highbrow, for example, is a reference to the structural changes of the forehead that we humans underwent to provide more space for our prefrontal cortices. It is thought that most of the unique qualities that humans possess are found or connected in some way with the expansion of the prefrontal cortex. This brain region is also important because the frontal lobes include areas of motor control as well. Proceeding anteriorly in the frontal lobes from the motor strip to the supplementary motor areas and the premotor cortices, we see the control of motor activity becoming more sophisticated (Figure 3). We also see that as the brain expanded and evolved anteriorly, the frontal lobes became more concerned with the cognitive control, timing, and duration of movement whereas the motor strip was an evolutionary advance giving humans greater gross voluntary motor control. The newer areas of the frontal lobe provide more precision and direction to 

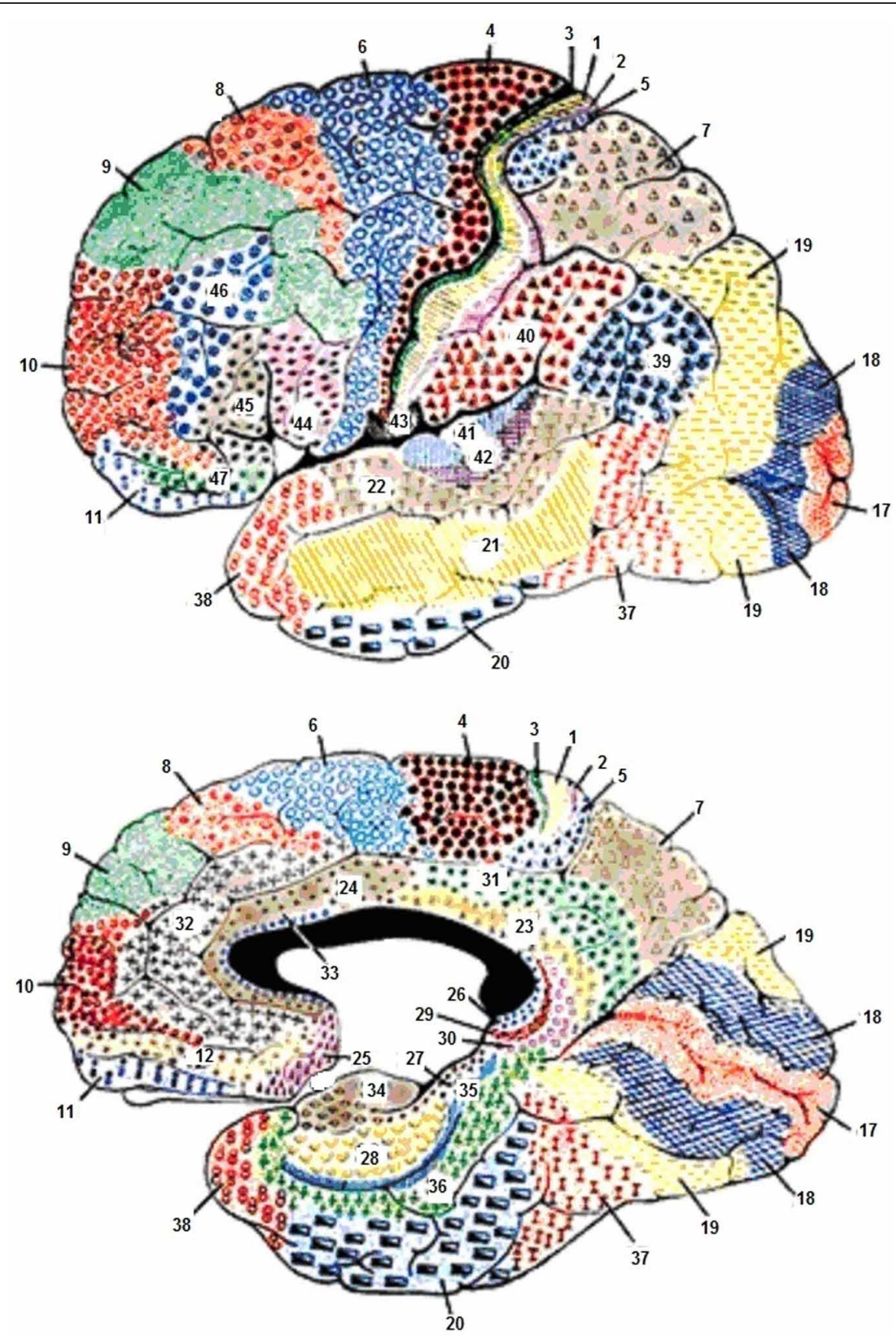

FIGURE 2 | Cytoarchitecture of the cortex with reference to the frontal lobes according to Broadmann. Areas 1, 2, and 3, Primary Somatosensory Cortex; Area 4, Primary Motor Cortex; Area 5, Somatosensory Association Cortex; Area 6, Pre-Motor and Supplementary Motor Cortex (Secondary Motor Cortex); Area 7, Somatosensory Association Cortex; Area 8, Includes Frontal eye fields; Area 9, Dorsolateral prefrontal cortex; Area 10, Frontopolar area (most rostral part of superior and middle frontal gyri); Area 11,

Orbitofrontal area (orbital and rectus gyri, plus part of the rostral part of the superior frontal gyrus); Area 12, Orbitofrontal area (used to be part of BA11, refers to the area between the superior frontal gyrus and the inferior rostral sulcus); Area 17, Primary Visual Cortex (V1); Area 18, Visual Association Cortex (V2); Area 19, (V3); Area 20, Inferior Temporal gyrus; Area 21, Middle Temporal gyrus; Area 22, Superior Temporal Gyrus, of which the rostral part participates to Wernicke's area; Area 23, Ventral Posterior cingulate cortex; Area 24,
Ventral Anterior cingulate cortex; Area 25, Subgenual cortex; Area 26, Ectosplenial area; Area 28, Posterior Entorhinal Cortex; Area 29,

Retrosplenial cingular cortex; Area 30, Part of cingular cortex; Area 31, Dorsal Posterior cingular cortex; Area 32, Dorsal anterior cingulate cortex; Area 34, Anterior Entorhinal Cortex (on the Parahippocampal gyrus); Area 35, Perirhinal cortex (on the Parahippocampal gyrus); Area 36, Parahippocampal cortex (on the Parahippocampal gyrus); Area 37, Fusiform gyrus; Area 38, Temporopolar area (most rostral part of the superior and middle temporal gyri); Area 39, Angular gyrus, part of Wernicke's area; Area 40, Supramarginal gyrus part of Wernicke's area; Areas 41 and 42, Primary and Auditory Association

Cortex; Area 43, Subcentral area (between insula and post/precentral gyrus); Area 44, pars opercularis, part of Broca's area; Area 45, pars triangularis Broca's area; Area 46, Dorsolateral prefrontal cortex; Area 47, Inferior prefrontal gyrus. 


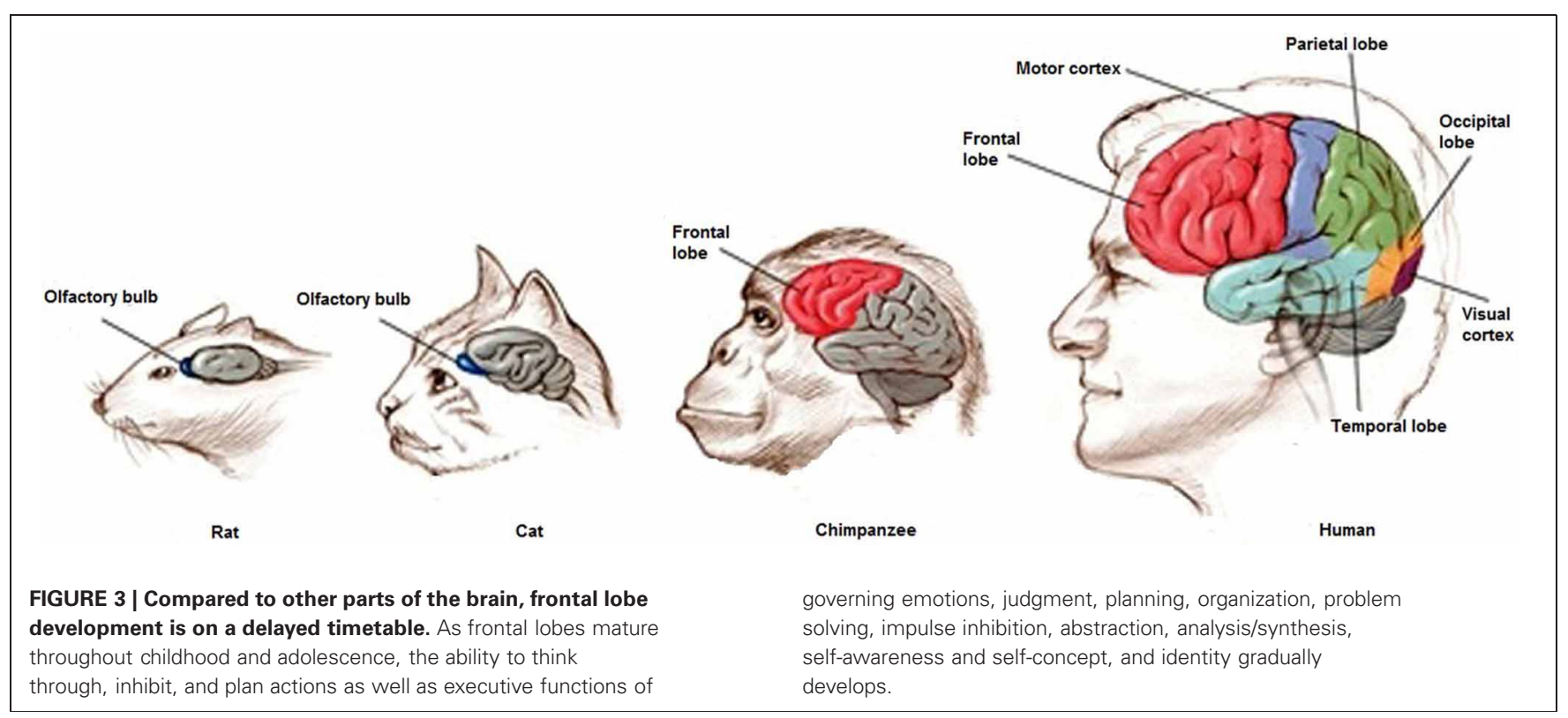

the movement. Eventually we see that the prefrontal cortex has little to do with the movement per se, but became largely concerned with the control of direction of the movement and the behavior that drives that movement.

\section{FRONTAL LOBES AND THE DEVELOPMENT OF GOAL-DIRECTED BEHAVIOR}

It is well-established that humans need normal frontal lobes to accomplish goals, make decisions, express creatively, and navigate through complex social situations (Chatterjee, 1998). The frontal lobes regulate goal-directed behavior, a hierarchy of reflexive movements, cross-temporal contingencies, approach and avoidance behavior, response inhibition, and perseveration. As we will see, all of the activities that the prefrontal cortex controls revolve around improvement of goal-directed behavior.

We hypothesize that the development of the human prefrontal cortices was a natural expansion of the evolutionarily earlier developed areas of the frontal lobe and that goal-directed movements and behavior provided for an expansion of those areas. The same regions of the human central nervous system that were already employed for better control, coordination, and timing of movements, expanded in parallel with the frontal cortex. The lateral portions of the cerebellum, for example, are more involved with the cognitive coordination and control of motor activity than with the control of the actual movement of muscles. The ventral lateral thalamus, linking the lateral cerebellum to the prefrontal cortex, is witness to the fact that these two areas evolved together. There must have developed a partnership between the cerebellum and the prefrontal cortex. The initial focus of the frontal lobes was the control of motor activity as it was for the cerebellum, but as the movements became more goal-directed, greater cognitive control over movement was necessitated.

The prefrontal cortex was required in higher organisms and in humans not just for more speed, precision, and coordination, but also for the provision of a control mechanism for memory of previous motor actions, projection of future movements, facilitation and inhibition of movement and the reaction or inhibition of reaction to stimuli; to know when to move toward prey or away from a predator. All of these involve higher cognitive control that all link to the emotion controlling limbic system which provides motivation, emotion in general, and aggression/temperament in particular, and autonomic self-regulation, the subject of free-will.

The links of frontal and prefrontal regions of the cortex to the limbic system, discussed in greater detail later, had as its main function the provision of a mechanism to either allow the organism to catch the prey, to run from a predator, or to seek a mate. The frontal lobes then in coordination with the cerebellum and basal ganglia have expanded beyond their control of movements and have evolved to control the behaviors that guide goal-directed movement and most of our basic actions, but driven in large measure by emotionality and its regulation, based upon approach and avoidance behavior.

It had been previously thought that the functions of the prefrontal cortex and its role in cognitive or emotional activities were separate from other motor areas of the frontal lobe (Luria, 1966). Therefore, motor functions of the frontal lobe did not necessarily relate to its non-motor function. However, it can be alternatively viewed that goal-directed behaviors are merely evolutionary expansions of goal-directed movements, and that all of the activities of the frontal lobe are variations and refinements of the same function. Achieving a goal is provided by the stimulation of the limbic system and therefore of emotionality. Therefore, temperament and frontal lobe development are associated and relate directly to the notion of free-will or choices made in the manifestation of goal-directed behavior.

\section{THE LONG DEVELOPMENT OF THE PREFRONTAL CORTEX, AND CRITICAL PERIODS FOR BEHAVIORAL INHIBITION AND THE DEVELOPMENT OF FREE-WILL}

Like other primates, humans are born with an immature brain. After birth, the cerebral cortex experiences a massive proliferation of synapses (synaptogenesis), followed by an extended pruning 
period (synaptic elimination). In the Rhesus Macaque-an old-world monkey whose brain development has been studied extensively-these developmental processes occur at the same rate in all cortical areas (Rakic et al., 1986). In contrast, analyses of human cortex across the life span (using autopsy tissue samples) reveal a different pattern. In humans, synaptogenesis reaches its peak in visual and auditory cortex within a few months after birth, but the increase in the number of synaptic junctions occurs much more slowly in the pre-frontal cortex (Huttenlocher and Dabholkar, 1997) (Figure 4). In the evolution of the human brain, there has thus been a shift from concurrent to heterochronous cortical development. The synaptic density of human PFC does not "catch up" with auditory cortex until the fourth year of life. Heterochronicity in human cortical development is also observed in measurements of dendritic arborization, regional metabolism (the extent of anabolic and catabolic processes within a brain region), and myelination (Chugani and Phelps, 1986).

As a result of this long period of prefrontal development, human children require an extended period to affect behavioral and cognitive control and thereby develop and exert free-will. The extended period of early child behavior is akin to adult patients with neurological PFC damage. Changes in both working memory capacity and the ability to produce behaviors that conflict with prepotent responses-two canonical frontal lobe functions - are linked to the maturation of PFC (e.g., Diamond and Doar, 1989). Furthermore, the extended immaturity of PFC may carry the cost of a longer period of vulnerability than that which occurs in more rapidly-developing cortical systems.

In the domain of emotion, crucial early experiences must occur for behavioral development to proceed on a normal track and for the brain systems underlying these behaviors to develop normally. This is achieved by neural plasticity. Greenough and colleagues have expanded on the notion of critical period and coined the term experience-expectant (Markham and Greenough, 2004), by which they mean a process by which synapses form after some minimal experience and is common to all members of the species thereby saving the genome the trouble of orchestrating and regulating all aspects of development. It is for this reason that the frontal lobes speak very well to the issue of the dynamics of change in early infant development. In general, we have a developmental pattern of temporary overproduction of synapses distributed within a relatively wide area during early development, followed by a subsequent retraction of synapses that had either not formed any or that had formed abnormal connections. The nature of neuronal connectivity is illustrated in Figure 5 below. The expected experience produces patterns of neuronal activity, targeting those synapses that will be selected for preservation. The assumption is that synaptic contacts are initially transient and require some type of confirmation for their continued survival. If such confirmation is not obtained, synapses will be retracted according to a developmental schedule or as a result of competition from confirmed synaptic connectivities (Black, 1998; Leisman and Koch, 2009).

In attempting to understand the neurosciences of free-will and in contradistinction to the above, Greenough and colleagues speak of experience-dependent neurogenesis. This is the process that optimizes the individual's adaptation to specific and possibly unique features of the environment e.g., learning. Thus, for any given instance, diverse information will be obtained and stored for use at a later time, giving rise to individual differences in a variety of cognitive domains, including temperament and
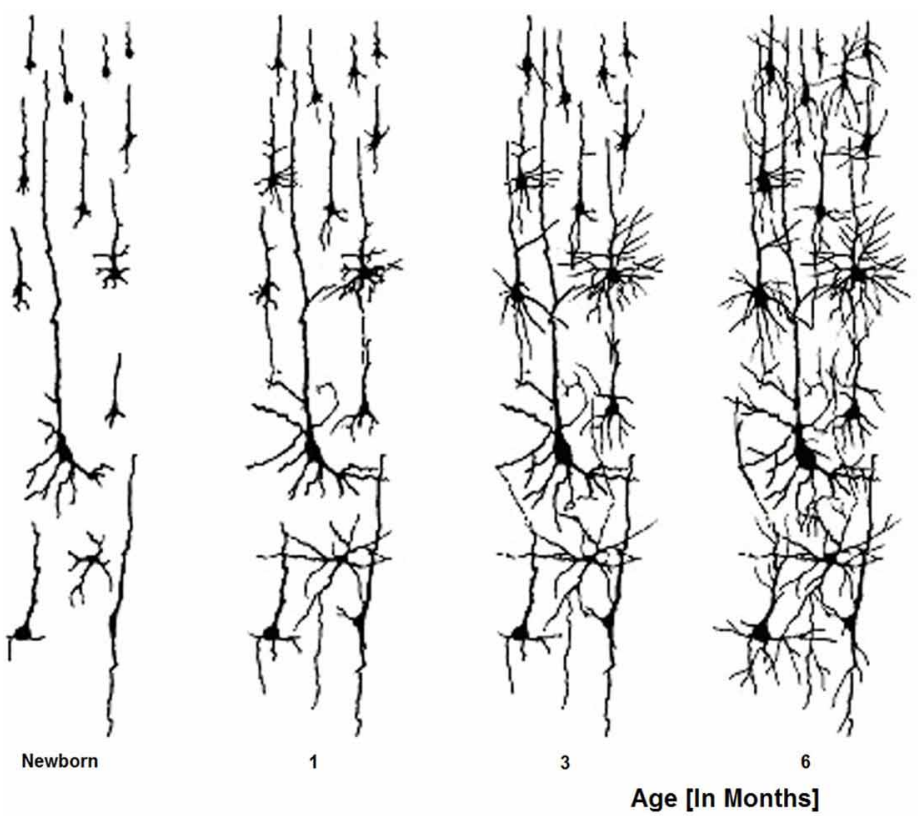

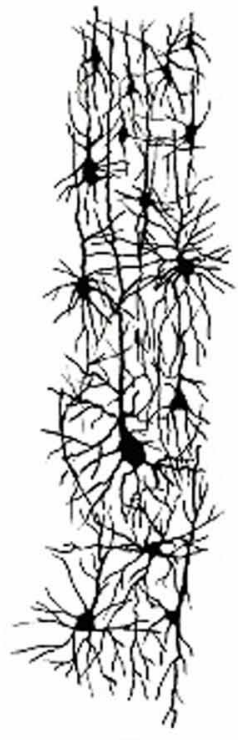

15

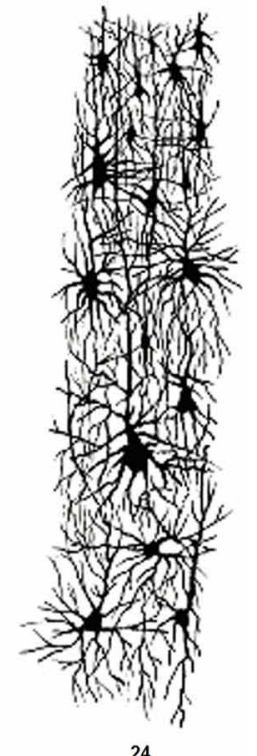

24

FIGURE 4 | Brain cells develop connections over the first two years of the infant's life. These connectivities are formed, altered, and actively sculpted over the first $20+$ years of life. 

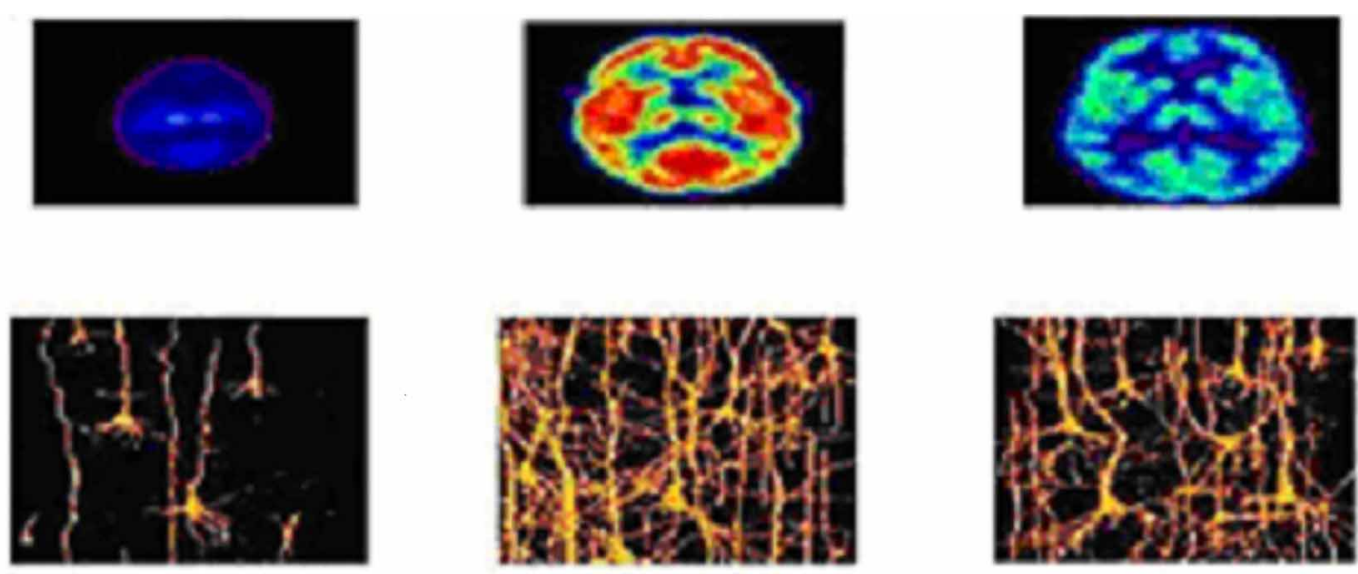

5 Days

6 Years

Adult

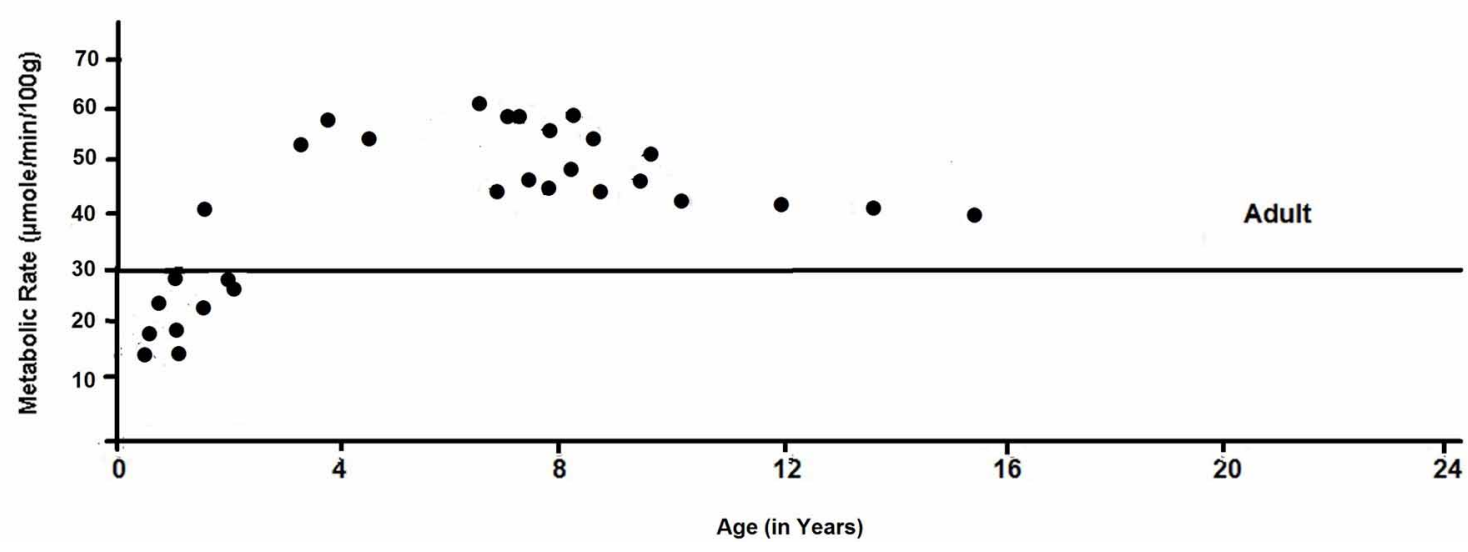

FIGURE 5 | Cerebral metabolic rate as a function of age. Elevated CMRGlc during 3-10 years corresponds to era of exuberant connectivity needed for energy needs of neuronal processes which is greater by a factor of 2 in childhood as compared to adults. PET shows relative glucose metabolic rate. We see the complexity of dendritic structures of cortical neurons consistent with expansion of synaptic connectivities and increases in capillary density in frontal cortex. emotionality. The fundamental difference between experienceexpectant and experience-dependent development is that the former applies in a similar fashion to all members of the species, whereas the latter applies to individual members, seen in normal emotional development in infancy.

The massive overproduction of synapses by the brain early in life followed postnatally by selective elimination of these exuberant connections has as its likely purpose the preparation of the nervous system for experience by the proliferation of connections on a sensory system-wide basis. Experience-related neuronal activity can then select functionally appropriate subsets of the abundant synaptic connections (represented in Figure 6). This period of excessive synaptogenesis is also correlated with a burst of brain metabolism and, at least in the monkey, with the onset of social interactions (Jacobs et al., 1995). The slow development of the frontal lobe allows the infant of the slow journey through circuit developments necessary for response inhibition, socialization, and emotional control. From the perspective we propose here, the adults' ability to control their responses allows them to mix and match correct and incorrect signs for the same things at different times, such that staying true to their probabilistic understanding leads them to produce noisy patterns of input. Absent these control abilities, young children will practice (and hence learn) only the most frequent of any alternate patterns they hear (Ramscar and Yarlett, 2007). This allows children to learn conventions from the output of parents who, because of the parents' ability to monitor and control their responses, may teach a correct response without the adults being able to master the correct responses themselves!

\section{CORTICAL AND LIMBIC INTERACTIONS IN THE DEVELOPMENT OF SELF-REGULATION AND FREE-WILL}

We can now explore in more detail the cortical and limbic interactions that are involved (represented in Figure 6) in the development of free-will. The literature has indicated that lesions in the dominant (right-handers) or the left hemisphere typically result in what has been termed "catastrophic reaction," for example, tears, despair, and anger. Damage to the right hemisphere, 


\section{The corticolimbic system}

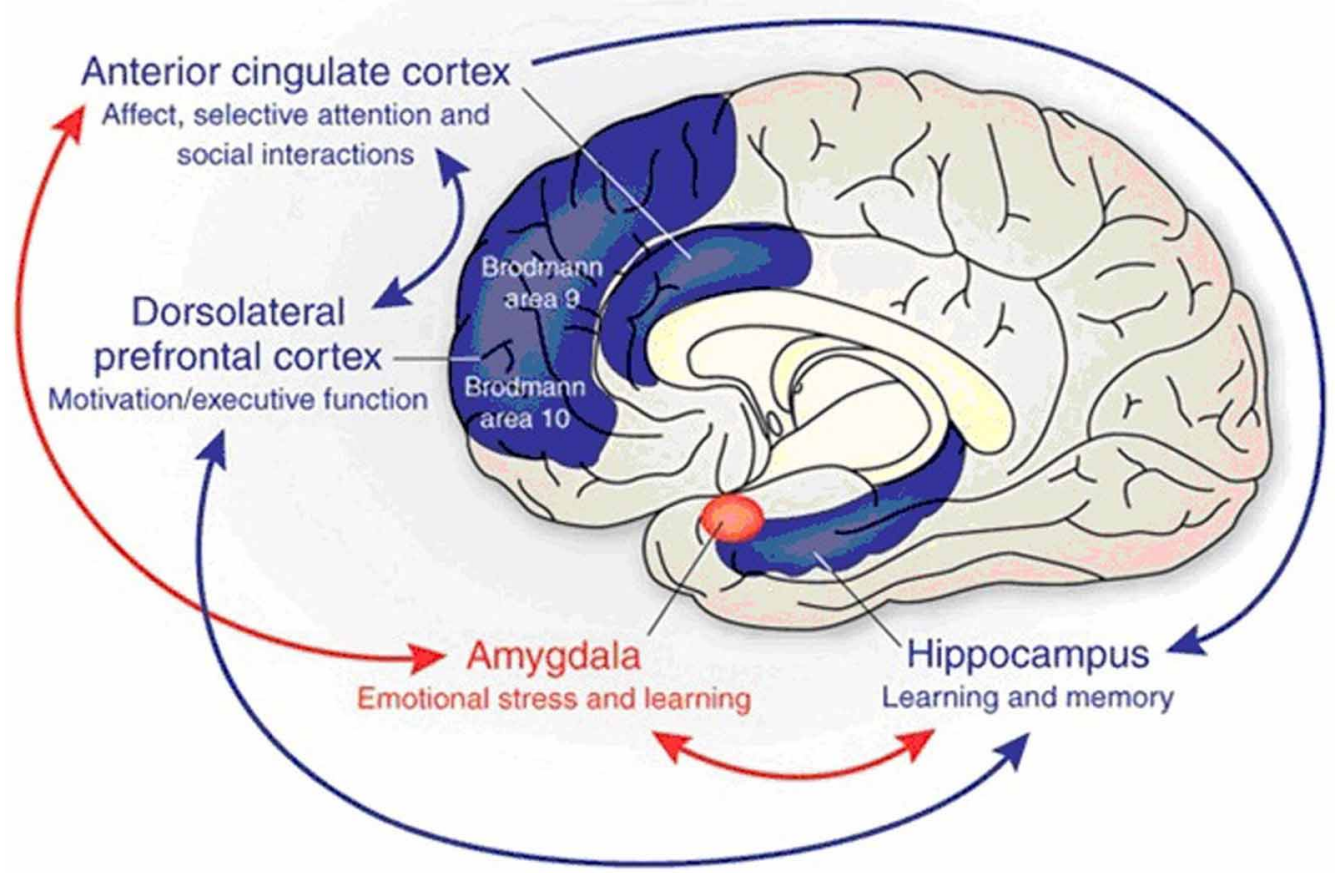

FIGURE 6 | The corticolimbic system consists of several brain regions that include the rostral anterior cingulate cortex, hippocampal formation, and basolateral amygdala. The anterior cingulate cortex has a central role in processing emotional experiences at the conscious level and selective attentional responses. Emotionally related learning is mediated through the interactions of the basolateral amygdala and hippocampal formation and motivational responses are processed through the dorsolateral prefrontal cortex (from Benes, 2010). which in most people is the minor hemisphere, is accompanied by indifference reactions such as unawareness, euphoria, or lack of concern (Goldstein, 1952; Gainotti, 1970, 1972). Therefore, the left and right hemispheres, based on this early research, were thought to result in opposite emotional tone with the left normally being more oriented toward a positive mood, whereas the right was oriented toward a more negative mood. Other studies have confirmed that pathologic crying occurs with left hemispheric lesions (Poeck, 1969; Rinn, 1984). It has been reported by Sackheim and associates (1982) that changes in affect following unilateral injury are due to disinhibition of contralateral cortical regions and not because of release of ipsilateral subcortical areas.

Anterior and posterior cortical regions appear to express different levels of control over the vertical hierarchy of subcortical centers (Robinson et al., 1984; Starkstein et al., 1988). It is further thought that the right hemisphere global conceptual skills may be critical to combining external and internal environmental information to achieve the integration of emotional experience (Safer and Leventhal, 1977). In other words, it is thought that the right hemisphere is able to access internal feelings that monitor an individual's internal state (Buck, 1985). It appears that the right hemisphere has greater interconnectivity between areas of the right hemisphere than the left hemisphere (Leisman and Ashkenazi, 1980; Iturria-Medina et al., 2011). This suggests that the specialized psychological abilities are due to its more diffuse interconnections, which provide it with a more dynamic and holistic integration across different sensory modalities.

It has been postulated that with lesions of the dorsolateral and dorsomedial prefrontal cortices, that exclude the frontal convexity, both slowness, indifference, apathy, and lack of initiative result. On the other hand, lesions of the orbito-frontal cortex appear to lead to disinhibition, lack of social constraint, hyperactivity, grandiose thinking, and euphoria (Smith et al., 2006; Volkow et al., 2011). There is also a lateral orbito-frontal circuit, projecting from the orbito-frontal cortex and connecting to different parts of the caudate and globus pallidus, which project to the thalamus and back to the orbito-frontal cortex. This circuit is thought to be involved in the control of inhibitory responses during learning and recognition tasks requiring frequent shifts of set developing during infancy. This may explain perseveration or repetitive compulsive behavior seen with damage to the orbito-frontal cortex and the perseveration seen in infancy. Another circuit, the anterior cingulate circuit, includes the ventral striatum, nucleus accumbens, and medio-dorsal nucleus of the thalamus. The hippocampus and entorhinal cortex are thought to send inputs to this circuit, which integrates information from the para-limbic association cortex.

Most researchers agree that approach and withdrawal are fundamental motivational behaviors, which are found at all levels of phylogeny. It has been postulated that the frontal lobes or anterior regions of the left and right hemispheres are specialized 
for approach and withdrawal behavior, respectively (Davidson, 1984, 1987, 1988; Davidson and Tomarken, 1989; Davidson et al., 1990). This is thought partly because the left frontal region has been noted as an important center for intention, self-regulation, and planning (Luria, 1973). This area is thought to be the region that produces behaviors that have been described as "free-will."

Volitional self-regulatory behavior is a significant part of the notion of free-will consistent with the view of most theorists on the topic. Ziaie (cf. Rothbart et al., 1992) performed a longitudinal study (at 3, 6.5, 10, and 13.5 months) in which the development of behaviors that serve to regulate distress were observed. The study was part of a larger longitudinal study in which infants were presented with auditory, visual, and tactile stimuli varying in novelty, intensity, and complexity, and their reactions videotaped. Because these episodes were designed to evoke emotional reactions, they proved to elicit a number of selfregulatory strategies as well. Behaviors that had been identified as self-regulatory in the literature were grouped together into larger functional categories of forms of self-regulation (Rothbart and Derryberry, 1981).

The larger categories included Active Avoidance (including specific behaviors of Arch Back, Arm Retraction, Leave Chair, Lean Away, Push Back, and Withdraw Hand), Orientation Toward Mother (Look Toward Mother, Lean Toward Mother, and Leave Chair Toward Mother), Disengagement of Attention (Gaze Aversion, Look Down, Look Away, Turn Head, and Look Toward Experimenter), Approach (Lean Forward, Reach, Point-Reach, and Inhibited Reach), Attack (Bang Toy, Pounding, and Push Toy Away), Body Self-Stimulation (Arm Movement, Banging, Body Movement, Kicking, and Repeated Hand Movement), Tactile Self-Soothing including Hand-Mouth Activity (Hand-Mouth, Mouthing), Touch Ear-Head, Clasp Hands, and Respiration (Heavy Breathing, Sigh, and Yawn).

Rothbart and Derryberry related self-regulatory behaviors to infant temperament by investigating relationships between infants' self-regulation at 13.5 months and emotionality as reported by the mother and observed in the laboratory. They found that by 13 months, the negative emotions of fear and "distress to limitations" were differentially related to patterns of self-regulation, with fear related to less active forms of coping, and "distress to limitations" to active attack.

\section{THE DEVELOPMENT OF MECHANISMS OF ATTENTION IN EMOTIONALITY AND SELF-REGULATORY BEHAVIOR: THE NEUROLOGICAL CAPACITY FOR SOCIAL INTERACTION MEDIATES THE DEVELOPMENT OF FREE-WILL}

Associated with approach-withdrawal developments is its relation to the development of attentional disengagement. The marked development of infants' orienting of attention in space undergoes marked development between two and six months of life, with changes related to infants' preferences for novel locations (Clohessy et al., 2001), their ability to disengage gaze from an external stimulus (Johnson et al., 2007), and their ability to anticipate the location of upcoming visual events (Haith et al., 1988). The development of infants' ability to disengage attention from one location so as to be able to move it to another and their ability to anticipate the location of future events is particularly important for the early self-regulation, self-control of emotion, and therefore free-will.

The development of these attentional abilities is in turn related to brain development (Johnson, 1990). Evidence from neuroanatomy indicates that only the deeper layers of primary visual cortex are supportive of organized activity in the newborn. During the first weeks of life, development of middle level lamina comes to support an inhibitory pathway to the superior colliculus. For a period of several weeks, this control system may inhibit disengagement when the infant is engaged at a visual location.

It is likely that this development is also responsible for the phenomenon of "obligatory attention" observed in young infants, where infants may look at a single location for extended periods, sometimes appearing to try to move their gaze but not being able to, and become distressed after a period of intent looking (Rothbart and Posner, 1985). By 4 months, development of both the parietal cortex (Posner and Peterson, 1990) and/or frontal eye field connections (Johnson, 1990) allows for more flexible disengagement of attention and greater self-regulation for the infant.

These early developments in attentional control are of interest to those who study social-emotional development because early changes in social interaction are related to these changing patterns of self-regulation. When caregiver and infant are observed interacting with one another in the vis a vis position (face-to-face), periods of extended visual orienting of the infant toward the mother seen at 6 and 13 weeks are followed by decreased orienting toward her by 6.5 months (Kaye and Fogel, 1980). In other studies, a shift of infant visual orienting to foci other than the mother has been observed by about 4 months of age (Kaye and Fogel, 1980; Cohn and Tronick, 1987). This change in infant orienting is often associated with the mother turning the infant away from the vis a vis position so that the child can more easily look around.

What we know then is that in self-regulation infants at three months infants already demonstrate ability to stimulate or soothe themselves, but engage in few approach behaviors. In comparison to three-month-old infants, six-month-old infants are more active stimulus seekers. They demonstrate greater use of organized patterns of motor behavior such as reaching, which showing further increases to 13 months of age. In comparison with their behavior at six months, 10-month-olds appear generally inhibited, showing less active self-stimulating behavior and more self-soothing. Increases in inhibitory capacity, self-soothing, and social communication thus appear to be hallmarks of development of self-regulation at 10 months. Thirteen-month-old infants, in comparison to 10-month-old infants, are more active at seeking stimulation, showed less self-soothing, more approach, fewer avoidance behaviors, and more self-stimulating behaviors than 10-month-old infants.

In their attentional regulation, they further increased their visual regard toward human beings as opposed to inanimate aspects of the environment. They also showed greater gestural communication in pointing and an increased ability to move objects away from themselves rather than moving themselves away from the objects. In general, a change from more palliative methods of self-regulation (e.g., clasping, mouthing) to more 
active coping, and a decrease over time in near receptor activity is seen. Overall, there were no changes in frequency of attentional disengagement from stimuli after six months, but older infants were more likely to redirect their attention toward their mothers. Major changes in disengagement occur between three and four months. The summary of the literature indicates that 3-13 months is a period of rapid development in self-regulatory behaviors, with little individual stability in their use other than in oral self-soothing, and with disengagement of attention continuing to be related to lower susceptibility to distress and consistent with the development of inhibitory systems (Rothbart et al., 1992). The absence or impairment of inhibitory systems results in well known disorders of disinhibition such as Tourette's syndrome (Ozonoff et al., 1998; Orth et al., 2005) or Obsessive-Compulsive Disorder (Moritza and von Mühlenen, 2005) which can be viewed as disorders of free-will and speak therefore to the neurological control of that function.

\section{DISCUSSION}

The nature of free-will as a subset of intentionality, and probabilistic and deterministic function, has indicated that human behavior is highly predictable which in turn, in part compromises a notion of a free-will. The literature supports the notion that age relates to the ability to progressively more effectively establish goals that are performed by fixed action patterns and that these FAPs produce outcomes and that these outcomes in turn modify choices (free-will) for which fixed action patterns need to be employed. Early goals require behaviors that need to be automated in terms of FAPs and that they in turn lead to an individual's goals being achieved or not; if not, then one can change behavior and that in turn is free-will.

Goals change with age based on experience which is similar to the way in which movement functions. We have supported the notion that the development of the human prefrontal cortices is a natural expansion of the evolutionarily earlier developed areas of the frontal lobe and that goal-directed movements and behavior including choice and free-will have provided for an expansion of those areas. The same regions of the human central nervous system that were already employed for better control, coordination, and timing of movement, expanded in parallel with the frontal cortex. The lateral portions of the cerebellum, for example, are more involved with the cognitive coordination and control of motor activity than with the control of the actual movement of muscles. The ventral lateral thalamus, linking the lateral cerebellum to the prefrontal cortex, is witness to the fact that these two areas evolved together. There must have developed a partnership between the cerebellum and the prefrontal cortex. The initial focus of the frontal lobes was the control of motor activity as it was for the cerebellum, but as the movements became more goal-directed, greater cognitive control over movement was necessitated and hence a necessity for "free-will."

The prefrontal cortex was required in higher organisms and in humans not just for more speed, precision, and coordination, but also for the provision of a control mechanism for memory of previous motor actions, projection of future movements, facilitation and inhibition of movement and the reaction or inhibition of reaction to stimuli; to know when to move toward prey or away from a predator. All of these involve higher cognitive control of the emotion controlling limbic system which provides motivation, emotion, aggression/temperament, and autonomic regulation.

The links of frontal and prefrontal regions of the cortex to the limbic system had as its main function the provision of a mechanism to either allow the organism to catch the prey, to run from a predator, or to seek a mate, or choice. The frontal lobes then, in coordination with the cerebellum and basal ganglia, have expanded beyond their control of movements and have evolved to control the behaviors that guide goal-directed movement and most of our basic actions, but driven in large measure by emotionality and its regulation, based upon approach and avoidance behavior.

A separate discussion involves not free-will per se but rather the responsibility over one's actions that results from free-will and these issues are oftentimes confused. We have seen that desires and actions are developmentally based on brain processes that are predicated on inhibition-facilitation and related to approach-avoidance dimensions and that are also predicated on the developing but integrated function of networks in the brain. All action and desire are driven by various brain processes. It can be questioned that while it is that the brain and nervous system are responsible for all human action and reaction, we human beings consider that we are responsible only when the system is intact but not when a tumor or dysfunction "causes" an act.

Sinnott-Armstrong and colleagues have examined individuals with brain tumors or other clearly neurologically based conditions including Tourette's syndrome, alien hand syndrome, addiction, psychopathy, and other mental illnesses, to obtain a better understanding of the notion of "responsibility." How people judge something to be morally right or wrong is a fundamental question of both the sciences and the humanities (Sinnott-Armstrong, 2011). Sinott-Armstrong has introduced a novel distinction between "moral deliberation," or the weighing of moral considerations, and the formation of a "moral verdict," or the commitment to one moral conclusion. Hemodynamic activity in the bilateral anterior insula and basal ganglia was found to be correlated with committing to the moral verdict "this is morally wrong" as opposed to "this is morally not-wrong," a finding that is consistent with research from economic decisionmaking. Using comparisons of deliberation-locked vs. verdictlocked analyses, it had also been demonstrated that hemodynamic activity in high-level cortical regions previously implicated in morality-including the ventro-medial prefrontal cortex, posterior cingulate cortex, and temporoparietal junction, was related primarily with moral deliberation as opposed to moral verdicts. The findings are consistent with individuals with psychopathologies who may possess moral judgment but impaired moral behavior.

Sinott-Armstrong and colleagues (Schaich et al., 2006) using fMRI have inferred the parts of the brain that activate when different types of moral judgments are made. Different cognitive functions and different brain mechanisms are involved when people make a moral judgment of an act causing harm (for example, a murder or theft) than when they judge an act seen as "impure" but harmless (such as special cases of cannibalism or consensual incest) or dishonest but harmless (such as some lies 
or broken promises). Each of these acts is termed "immoral," but at a physical level, morality is not a unified concept yet free-will seems to be. With conscious free-will and its bearing on legal and moral responsibility, we normally excuse people whose acts are not caused by their conscious choices, such as sleepwalkers who murder and those with neoplasms who have committed crimes.

Surprisingly, recent research suggests that conscious choice plays a smaller role in our actions than most people assume. In particular, it often comes after brain activity that initiates bodily movements, and many researchers conclude that the conscious choice does not cause the movement (cf. Melillo and Leisman, 2009a,b). That conclusion raises the disturbing questions of whether and how we can ever really be responsible for anything. Known for a while is the necessity to automate as much as possible which arises from the need to reduce information overload on the nervous system due to its relatively limited capacity for instantaneous information processing (Leisman, 1976; Melillo and Leisman, 2009a,b). This is precisely the reason why we neither look nor need to do so when walking down a flight of stairs. The issue of responsibility is both scientific and moral. Freedom exists within a deterministic universe. Our knowledge surrounding consciousness is incomplete, and it may ultimately transpire that brain activity does not cause conscious

\section{REFERENCES}

Benes, F. M. (2010). Amygdalocortical circuitry in schizophrenia: from circuits to molecules. Neuropsychopharmacology 35, 239-257.

Black, J. E. (1998). How a child builds its brain: some lessons from animal studies of neural plasticity. Prev. Med. 27, 168-171.

Buck, R. (1985). Prime theory: an integrated view of motivation and emotion. Psychol. Rev. 92, 389-413.

Buss, A. H., and Plomin, R. (1975). A Temperament Theory of Personality Development. New York, NY: Wiley.

Buss, A. H., and Plomin, R. (1986). "The EAS approach to temperament," in The Study of Temperament: Changes, Continuities and Challenges Plomin, eds R. Plomin and J. Dunn (Hillsdale, NJ: Lawrence Erlbaum), 67-77.

Chatterjee, A. (1998). Feeling frontal dysfunction: facilitory paratonia and the regulation of motor behavior. Neurology 51, 937-939.

Chugani, H. T., and Phelps, M. E. (1986). Maturational changes in cerebral function in infants determined by 18FDG positron emission tomography. Science 231, 840-843.

Clohessy, A. B., Posner, M. I., and Rothbart, M. K. (2001). Development of the functional visual field. Acta Psychol. (Amst.) 106, 51-68.

Cohn, J. E., and Tronick, E. Z. (1987). Mother-infant face-to-face interaction: the sequencing of dyadic states at 3, 6, and 9 months. Dev. Psychol. 23, 68-77.

Davidson, R. J. (1984). "Affect, cognition and hemispheric specialization," in Emotion, Cognition, and Behavior, eds C. E. Izard, J. Kagan, and R. Zajonc (New York, NY: Cambridge University Press), 320-365.

Davidson, R. J. (1987). "Cerebral asymmetry and the nature of emotion: implications for the study of individual differences and psychopathology," in Cerebral Dynamics, Laterality and Psychopathology, eds R. Takahashi, P. Flor-Henry, J. Gruzelier, and S. Niwa (New York, NY: Elsevier), 71-83.

Davidson, R. J. (1988). EEG measures of cerebral asymmetry: conceptual and methodological issues. Int. J. Neurosci. 39, 71-89.

Davidson, R. J. (2000). Affective style, psychopathology, and resilience: brain mechanisms and plasticity. Am. Psychol. 55, 1196-1214.

Davidson, R. J., Ekman, P., Saron, C., Senulis, J., and Friesen, W. V. (1990). Approach/withdrawal and cerebral asymmetry: emotional expression and brain physiology: I. J. Pers. Soc. Psychol. 58, 330-341.

Davidson, R. J., and Fox, N. A. (1989). Frontal brain asymmetry predicts infants' response to maternal separation. J. Abnorm. Psychol. 98, 127-131.

decision-making or vice versa, but rather a variety of cognitive processes occurring almost simultaneously (Leisman and Melillo, 2012).

In conclusion it has been suggested by Perez-Velazquez (2012, Pers. communication) that Michael Gazzaniga's (2005) views on the subject bears revisiting, when he stated, "... Brains are automatic, rule-governed, determined devices, while people are personally responsible agents, free to make their own decision. Just as traffic is what happens when physically determined cars interact, responsibility is what happens when people interact. Personal responsibility is a public concept. It exists in a group, not in an individual. If you were the only person on earth, there would be no concept of personal responsibility. Responsibility is a concept you have about other people's actions and they have about yours. Brains are determined; people follow rules when they live together and out of that interaction arises the concept of freedom of action ... They exists only in the relationships that exist when automatic brains interact with other automatic brains."

\section{ACKNOWLEDGMENTS}

This work is supported by a grant from the Ministry of Science under Kamea Dor-B to Gerry Leisman and from Help the Hope to Gerry Leisman and Calixto Machado.

Davidson, R. J., and Tomarken, A. J. (1989). "Laterality and emotion: an electrophysiological approach," in Handbook of Neuropsychology, eds F. Boiler and J. Grafman (Amsterdam: Elsevier), 419-441.

Diamond, A., and Doar, B. (1989). The performance of human infants on a measure of frontal cortex function, the delayed response task. Dev. Psychobiol. 22, 271-294.

Fox, N. A., Henderson, H. A., Rubin, K. H., Calkins, S. D., and Schmidt, L. A. (2001). Continuity and discontinuity of behavioral inhibition and exuberance: psychophysiological and behavioral influences across the first four years of life. Child Dev. $72,1-21$.

Gainotti, G. (1970). Emotional behavior of patients with right and left brain damage in neuropsychological test conditions. Arch. Psicol. Neurol. Psichiatr. 31, 457-480.

Gainotti, G. (1972). Emotional behavior and hemispheric side of the lesion. Cortex 8, 41-55.

Gazzaniga, M. S. (2005). The Ethical Brain: The Science of our Moral Dilemmas. Washington, DC: Dana Press.

Goldsmith, H. H., Buss, A. H., Plomin, R., Rothbart, M. K., Thomas, A., Chess, S., Hinde, R. A., McCall, R., and Roundtable, B. (1987). What is temperament? Four approaches. Child Dev. 58, 505-529.
Goldstein, K. (1952). The effect of brain damage on the personality. Psychiatry 15, 245-260.

Haith, M. M., Hazan, C., and Goodman, G. S. (1988). Expectation and anticipation of dynamic visual events by 3.5-monthold babies. Child Dev. 59, 467-479.

Henderson, H. A., Fox, N. A., and Rubin, K. H. (2001). Temperamental contributions to social behavior: the moderating roles of frontal EEG asymmetry and gender. J. Am. Acad. Child Adolesc. Psychiatry 40, 68-74.

Huttenlocher, P. R., and Dabholkar, A. S. (1997). Regional differences in synaptogenesis in human cerebral cortex. J. Comp. Neurol. 387, 167-178.

Iturria-Medina, Y., Pérez Fernández, A., Morris, D. M., Canales-Rodríguez, E. J., Haroon, H. A., García Pentón, L., Augath, M., Galán García, L., Logothetis, N., Parker, G. J., and Melie-García, L. (2011). Brain hemispheric structural efficiency and interconnectivity rightward asymmetry in human and nonhuman primates. Cereb. Cortex 21, 56-67.

Jacobs, B., Chugani, H. T., Allada, V., Chen, S., Phelps, M. E., Pollack, D. B., and Raleigh, M. J. (1995). Developmental changes in brain metabolism in sedated rhesus macaques and vervet monkeys revealed by positron emission 
tomography. Cereb. Cortex 5, Luria, A. R. (1966). Higher Cortical 222-233.

Johnson, M. H. (1990). Cortical maturation and the development of visual attention in early infancy. $J$. Cogn. Neurosci. 2, 81-95.

Johnson, S. C., Ok, S. J., and Luo, Y. (2007). The attribution of attention: 9-month-olds' interpretation of gaze as goal-directed action. Dev. Sci. 10, 530-537.

Kandel, E. R. (1999). Biology and the future of psychoanalysis: a new intellectual framework for psychiatry revisited. Am. J. Psychiatry 156, 504-524.

Kaye, K., and Fogel, A. (1980). The temporal structure of face to face communication between mothers and infants. Dev. Psychol. 16, 454-464.

Kennard, M. A., Viets, H. R., and Fulton, J. F. (1934). The syndrome of the premotor cortex in man: impairment of skilled movements, forced grasping, spasticity, and vasomotor disturbance. Brain 57, 69-84.

Kochanska, G. (1995). Children's temperament, mothers' discipline, and security of attachment: multiple pathways to emerging internalization. Child Dev. 66, 597-615.

Leisman, G. (1976). "The role of visual processes in attention and its disorders," in Basic Visual Processes and Learning Disability, ed G. Leisman (Springfield, IL: Charles C. Thomas), 7-123.

Leisman, G., and Ashkenazi, M. (1980). Aetiological factors in dyslexia: IV. Cerebral hemispheres are functionally equivalent. Int. J. Neurosci. 11, 157-164.

Leisman, G., and Koch, P. (2009). Networks of conscious experience: computational neuroscience in an understanding of life, death, and consciousness. Rev. Neurosci. 20, 151-176.

Leisman, G., and Melillo, R. (2006). "Cortical asymmetry and learning efficiency: a direction for the rehabilitation process," in Learning Disabilities: New Research, ed S. V. Randall (Hauppauge, NY: Nova), $1-24$.

Leisman, G., and Melillo, R. (2012). "The development of the frontal lobes in infancy and childhood: asymmetry and the nature of temperament and affect," in Frontal Lobe: Anatomy, Functions and Injuries, ed A. Cavanna (Hauppauge, NY: Nova) (in press). Basic Books.

Luria, A. R. (1973). The Working Brain: An Introduction to Neuropsychology. New York, NY: Basic Books.

Markham, J. A., and Greenough, W. T. (2004). Experience-driven brain plasticity: beyond the synapse. Neuron Glia Biol. 1, 351-363.

Melillo, R., and Leisman, G. (2009a). Neurobehavioral Disorders of Childhood: An Evolutionary Perspective. New York, NY: Springer Science.

Melillo, R., and Leisman, G. (2009b). Autism spectrum disorder as functional disconnection syndrome. Rev.

Moritza, S., and von Mühlenen, A. (2005). Inhibition of return in patients with obsessive-compulsive disorder. J. Anxiety Disord. 19, 117-126.

Orth, M., Amann, B., Robertson, M. M., and Rothwell, J. C. (2005). Excitability of motor cortex inhibitory circuits in Tourette syndrome before and after single dose Nicotine. Brain 128, 1292-1300.

Ozonoff, S., Strayer, D. L., McMahon, W. M., and Filloux, F. (1998). Inhibitory deficits in Tourette syndrome: a function of comorbidity and symptom severity. J. Child Psychol. Psychiatry 39, 1109-1118.

Pluess, M., and Belsky, J. (2011). Prenatal programming of postnatal plasticity? Dev. Psychopathol. 23, 29-38.

Poeck, K. (1969). "Pathophysiology of emotional disorders associated with brain damage," in Handbook of Clinical Neurology, Vol. 3, eds P. J. Vinken and G. W. Bruyn (New York, NY: Elsevier), 343-376.

Porges, S. W., and Furman, S. A. (2011). The early development of the autonomic nervous system provides a neural platform for social behavior: a polyvagal perspective. Infant Child Dev. 20, 106-118.

Posner, M. L., and Peterson, S. E. (1990). The attention system of the human brain. Annu. Rev. Neurosci. 13, 25-42.

Rakic, P., Bourgeois, J. P., Eckenhoff, M. F., Zecevic, N., and Goldman-Rakic, P. S. (1986). Concurrent overproduction of synapses in diverse regions of the primate cerebral cortex. Science 232, 232-235.

Ramscar, M., and Yarlett, D. (2007). Linguistic self-correction in the absence of feedback: a new Neurosci. 20, 111-132. approach to the logical problem of language acquisition. Cogn. Sci. 31, 927-960.

and intention as factors in moral judgments: an fMRI investigation. J. Cogn. Neurosci. 18, 803-817.

Rinn, W. E. (1984). The neuropsychology of facial expression: a review of the neurological and psychological mechanisms for producing facial expressions. Psychol. Bull. 95, 52-77.

Robinson, R. G., Kubos, K. L., Starr, L. B., Rao, K., and Price, T. R. (1984). Mood disorders in stroke patients: importance of location of lesion. Brain 107, 81-93.

Rothbart, M. K. (1989). "Biological process of temperament," in Temperament in Childhood, eds G. A. Kohnstamm, J. E. Bates, M. K. Rothbart (Chichester, UK: Wiley), 77-110.

Rothbart, M. K., and Bates, J. E. (1998). “Temperament," in Handbook of Child Psychology: vol 3. Social Emotional, and Personality Development (Fifth ed.), eds W. Damon (Series Ed.), and N. Eisenberg (Vol. Ed.), (New York, NY: Wiley), 105-176.

Rothbart, M. K., and Derryberry, D. (1981). "Development of individual differences in temperament," in Advances in Developmental Psychology, Vol. 1, eds M. E. Lamb and A. Brown (Hillsdale, NJ: Erlbaum), 37-86.

Rothbart, M. K., and Posner, M. I. (1985). "Temperament and the development of self-regulation," in Neuropsychology of Individual Differences: A Developmental Perspective, eds L. H. Hartlage and C. F. Telzrow (New York, NY: Plenum), 93-124

Rothbart, M. K., Ziaie, H., and O'Boyle, C. G. (1992). Self-regulation and emotion in infancy. New Dir. Child Dev. 55, 7-23.

Rowe, D. C., and Plomin, R. (1977). Temperament in early childhood. J. Pers. Assess. 41, 150-156.

Sackeim, H. A., Greenberg, M. S., Weiman, A. L., Gur, R. C. Hungerbuhler, J. P., and Geschwind, N. (1982). Hemispheric asymmetry in the expression of positive and negative emotions: neurologic evidence. Arch. Neurol. 39, 210-218.

Safer, M. A., and Leventhal, H. (1977). Ear differences in evaluating emotional tone of voice and verbal content. J. Exp. Psychol. Hum. Percept. Perform. 3, 75-82.

Schaich, B. J., Hynes, C., Van Horn, J., Grafton, S., and Sinnott-Armstrong, W. (2006). Consequences, action,
Sinnott-Armstrong, W. P. (2011). Neurolaw and consciousness detection. Cortex 47, 1246-1247.

Smith, J. R., Lee, G. P., Fountas, K., King, D. W., and Jenkins, P. D. (2006). Intracranial stimulation study of lateralization of affect. Epilepsy Behav. 8, 534-541.

Starkstein, S. E., and Robinson, R. T. (1988). Comparisons of patients with and without post-stroke major depression matched for size and location of lesion. Arch. Gen. Psychiatry 45, 247-252.

Touwen, B. C. (1971). A study on the development of some motor phenomena in infancy. Dev. Med. Child Neurol. 13, 435-446.

Volkow, N. D., Tomasi, D., Wang, G. J., Fowler, J. S., Telang, F., Goldstein, R. Z., Alia-Klein, N., Woicik, P., Wong, C., Logan, J., Millard, J., and Alexoff, D. (2011). Positive emotionality is associated with baseline metabolism in orbitofrontal cortex and in regions of the default network. Mol. Psychiatry 16, 818-825.

Wiemann, M., Frede, S., Tschentscher, F., Kiwull-Schöne, H., Kiwull, P., Bingmann, D., Brinkmann, B., and Bajanowski, T. (2008). NHE3 in the human brainstem: implication for the pathogenesis of the sudden infant death syndrome (SIDS)? $A d v$. Exp. Med. Biol. 605, 508-513.

Conflict of Interest Statement: The authors declare that the research was conducted in the absence of any commercial or financial relationships that could be construed as a potential conflict of interest.

Received: 15 March 2012; accepted: 31 May 2012; published online: 27 June 2012.

Citation: Leisman G, Machado C, Melillo $R$ and Mualem $R$ (2012) Intentionality and "free-will" from a neurodevelopmental perspective. Front. Integr. Neurosci. 6:36. doi: 10.3389/ fnint.2012.00036

Copyright (C) 2012 Leisman, Machado, Melillo and Mualem. This is an openaccess article distributed under the terms of the Creative Commons Attribution Non Commercial License, which permits non-commercial use, distribution, and reproduction in other forums, provided the original authors and source are credited. 